\title{
The ablation of gas clouds by blazar jets
}

\author{
Jonathan Heil ${ }^{1}$ and Michael Zacharias ${ }^{1,2, \star}$ \\ ${ }^{1}$ Ruhr Astroparticle and Plasma Physics Center (RAPP Center), Insitut für Theoretische Physik IV, Ruhr-Universität Bochum, \\ 44780 Bochum, Germany \\ e-mail: hejo@tp4.rub.de \\ 2 Centre for Space Research, North-West University, Potchefstroom 2520, South Africa \\ e-mail: mzacharias.phys@gmail.com
}

Received 11 March 2020 / Accepted 20 July 2020

\section{ABSTRACT}

\begin{abstract}
Context. Flaring activity in blazars can last for vastly different timescales, and it may be the result of density enhancements in the jet flow that result from the intrusion of an interstellar cloud into the jet.

Aims. We investigate the lightcurves expected from the ablation of gas clouds by the blazar jet under various cloud and jet configurations.

Methods. We derived the semi-analytical formulae describing the ablation process of a hydrostatic cloud and performed parameter scans of artificial set-ups over both cloud and jet parameter spaces. We then used parameters obtained from measurements of various cloud types to produce lightcurves of these cloud examples.

Results. The parameter scans show that a vast zoo of symmetrical lightcurves can be realized. Both cloud and emission region parameters significantly influence the duration and strength of the flare. The scale height of the cloud is one of the most important parameters as it determines the shape of the lightcurve. In turn, important cloud parameters can be deduced from the observed shape of a flare. The example clouds result in significant flares lasting for various timescales.
\end{abstract}

Key words. radiation mechanisms: non-thermal - galaxies: active - galaxies: jets - gamma rays: galaxies

\section{Introduction}

Relativistic jets produce a vast range of flaring activity that can last from a few minutes, as in the case of gamma-ray bursts, up to months or years, as in the case of active galactic nuclei (AGNs). Jets and their flares are most easily studied in blazars, where the jet of the active galaxy points toward Earth (Urry \& Padovani 1995) and persists over long timescales. Blazars, as all relativistic jets, exhibit flares that substantially differ in duration and evolution (e.g., Zacharias 2018, and references therein). A common scenario involves a change in the plasma flow across a shock within the jet (Marscher \& Gear 1985). However, the details of the variation of the plasma parameters, which fit the lightcurves, have to be set arbitrarily. Most easily, a variation in the plasma density can account for a flare, and the modulation in the injection results in the specific lightcurve profile. Natural sources of density fluctuations are either a variable particle injection process at the base of the jet, which is possibly coupled to variations in the accretion disk, or through the pickup of material while the jet moves through the host galaxy. For the latter process, material can be supplied by the interstellar gas, stellar astrospheres, supernova remnants, etc.

Zacharias et al. $(2017,2019)$ used the injection of pick-up material to explain the bright and long-lasting flare of the blazar CTA 102. In their model, a gas cloud approached the jet and was subsequently ablated. In their picture, the cloud slowly intruded into the jet, implying a smoothly varying injection of particles. The number of particles injected into the jet flow depended on the geometry and the density structure of the cloud; few particles were injected in the beginning and at the end of the process

\footnotetext{
^ Now at: Laboratoire Univers et Théories, Observatoire de Paris, Université PSL, CNRS, Université de Paris, 92190 Meudon, France.
}

and most particles were injected when the center of the cloud intruded the jet.

The successful reproduction of the CTA 102 flare is reassuring. However, in order to understand the full potential of the model, it is necessary to study the influence of various parameters from both the cloud and jet on the lightcurve. Therefore in this paper, we revisit the cloud-ablation model from a theoretical point-of-view. We first explore the requirements of cloud and jet parameters for the ablation to proceed, followed by deriving the time evolution of the particle injection. This is described in Sect. 2 along with a general discussion of the model. We then study the lightcurves of both theoretical (Sect. 3) and a few exemplary, real (Sect. 4) clouds. The results are discussed in Sect. 5.

\section{The cloud ablation model}

Clouds, such as those of the broad-line region (BLR) but also stars and their astrospheres, penetrating the relativistic jet of an AGN, have been explored in various applications. Generally, the time-dependent intrusion of the cloud into the jet results in a similar time-dependency of the particle injection into the jet flow. The destruction of the cloud is a consequence of the relativistic jet flow and the associated ram pressure.

The timescale of the ablation process is governed by the speed of the shock that is formed in the cloud as it starts penetrating the jet, that is, the time the shock needs to cross the cloud. For a rough estimate, one can assume that the shock speed is roughly $c / 4^{1}$, where $c$ is the speed of light. The time for the

\footnotetext{
1 In the downstream frame, the speed of a strong shock is $v_{s}=u_{1} / 4$. The upstream speed is $u_{1} \sim c$, i.e., the speed of the jet.
} 


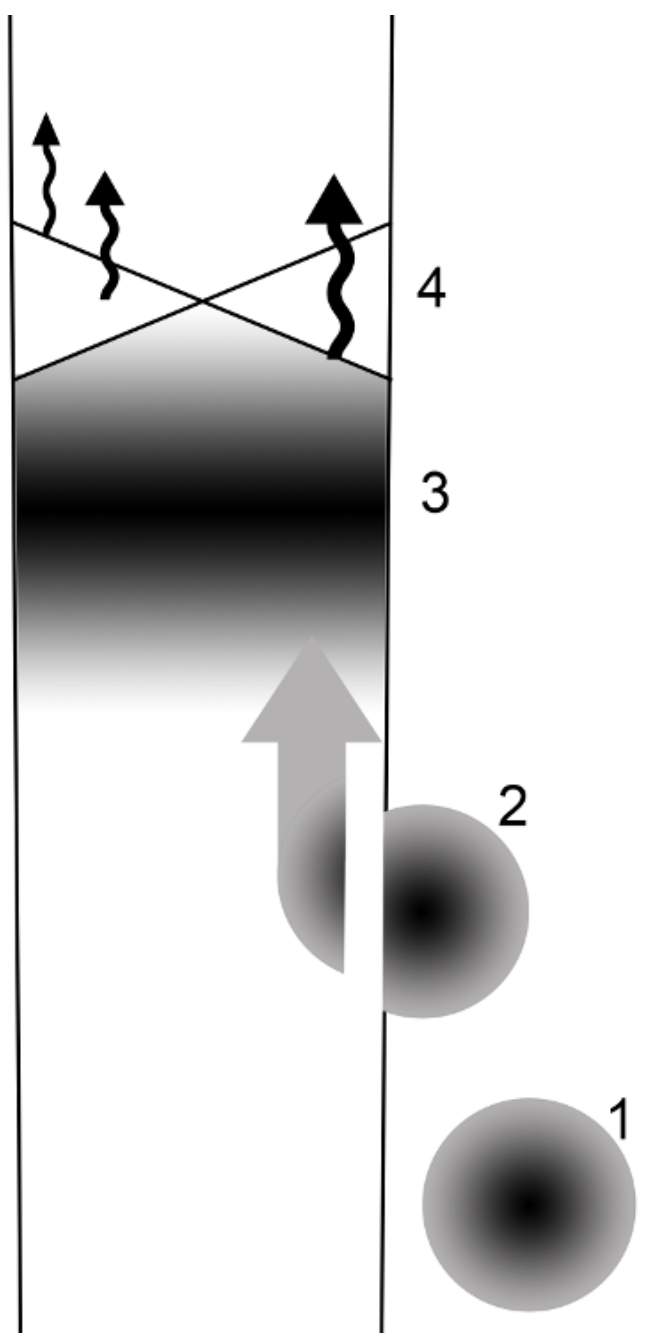

Fig. 1. Sketch of the ablation process (not to scale). (1) The cloud approaches the jet and (2) is ablated slice-by-slice while entering the jet. (3) The ablated cloud material is mixed into the jet flow, resulting in a specific density enhancement. (4) At a downstream shock, the particles are accelerated to nonthermal energies and they radiate.

shock to pass the cloud is $t_{s}=8 R / c$, with the cloud radius $R$. If the intrusion time of the cloud into the jet $t_{\text {in }}=2 R / v$, where $v$ is the cloud speed, is shorter than $t_{s}$, the cloud may penetrate deep into the jet before the shock has crossed the object. In this case, the cloud material would be shocked in one instance, resulting in a violent burst of particles and radiation (Araudo et al. 2010). This provides the ingredients for fast flares (e.g., Barkov et al. 2012a,b).

On the other hand, if $t_{s}<t_{\text {in }}$, the shock mostly interacts with the subvolume of the cloud that has already penetrated the jet. In turn, the ablation process is gradual (Zacharias et al. 2017) as depicted in Fig. 1. Simulations by Perucho et al. (2017) involving a jet-star interaction suggest that the ablation of the astrosphere already begins in the transition layer at the edge of the jet and proceeds while the star continues to penetrate the jet. The ablated material is mixed into the jet flow, where it is accelerated to the jet's bulk speed. The acceleration to nonthermal energies may take place at a downstream shock, such as a recollimation shock (Marscher et al. 2008). These types of shocks are ubiquitous in blazar jets (Jorstad et al. 2017). Radiative processes (Torres-Albà \& Bosch-Ramon 2019) may occur close to the shock region. At this point, we ignore the possibility that shock waves are formed in the jet through the cloud intrusion itself (Bosch-Ramon 2012; Perucho et al. 2017). The potential variations in shock strength and size throughout the jetcloud interaction cannot be easily quantified and require indepth (magneto-)hydrodynamic (MHD) simulations, which are beyond the scope of this paper.

Following the gradual ablation and injection of particles into the jet flow, the subsequent acceleration of the particles and emission of radiation proceed similarly. A resulting flare evolves smoothly and is probably symmetric in time ${ }^{2}$, as the change in the particle injection dominates the lightcurve, while other jet parameters - such as the shock radius - probably remain fixed.

In this paper, we focus on the second scenario, where a cloud enters the jet and is slowly ablated, leading to a long-lasting flux enhancement. In order to calculate the amount of ablated material at a given instant in time, the cloud's geometry and density structure are required. While the geometry has a significant influence on the ablated volume, we assume only a spherical geometry for ease of computation. Furthermore, we only consider leptonic radiation processes. While the hadronic components of the cloud are carried along in the jet, we assume that they remain energetically cold and do not participate in the radiation processes, which is a common, albeit debated assumption in blazar modeling (e.g., Böttcher 2007; Ghisellini et al. 2011; Böttcher et al. 2013; Zdziarski \& Böttcher 2015; Cerruti et al. 2015, 2017; H.E.S.S. Collaboration 2019). Below, we describe the cloud's density structure, the jet condition to ablate the cloud, and the resulting injection function.

\subsection{Cloud density}

An isothermal cloud is bound by its own gravitational pull. If we ignore any external forces that may shape it into different structures, the cloud is spherically symmetric with a radial coordinate $r$. As discussed in Zacharias et al. (2017), the cloud's density structure $n(r)$ is defined by the equation of hydrostatic equilibrium

$k_{\mathrm{B}} T \frac{\mathrm{d} n(r)}{\mathrm{d} r}=-4 \pi \frac{G m_{p}^{2} n(r)}{r^{2}} \int_{0}^{r} \mathrm{~d} \bar{r} \bar{r}^{2} n(\bar{r})$

with the temperature $T$, Boltzmann's constant $k_{\mathrm{B}}$, the proton mass $m_{\mathrm{p}}$, and the gravitational constant $G$. We have assumed that the cloud predominantly consists of hydrogen.

With some manipulations of Eq. (1), one reaches the LaneEmden equation, which does not provide an analytical solution in this case. However, from the asymptotic solution $n \propto r^{-2}$, as well as the boundary conditions $n(0)<\infty$ and $\mathrm{d} n /\left.\mathrm{d} r\right|_{r=0}=$ 0 , one can derive a reasonable approximation (Zacharias et al. 2017; Banda-Barragán et al. 2018):

$n(r)=\frac{n_{0}}{1+\left(r / r_{0}\right)^{2}}$,

with the central density $n_{0}$, and the scale height

$$
\begin{aligned}
r_{0} & =\sqrt{\frac{2 k_{\mathrm{B}} T}{4 \pi G m_{p}^{2} n_{0}}}=\sqrt{\tilde{c} \frac{T}{n_{0}}} \\
& =4 \times 10^{12}\left(\frac{T}{140 \mathrm{~K}}\right)^{1 / 2}\left(\frac{n_{0}}{10^{15} \mathrm{~cm}^{-3}}\right)^{-1 / 2} \mathrm{~cm},
\end{aligned}
$$

2 We note that this remark concerns the long-term behavior of the flare. On shorter timescales, substructures in the cloud or instabilities in the jet due to the ablation process may still trigger shorter spikes in the lightcurve. 
with $\tilde{c}:=2 k_{\mathrm{B}} /\left(4 \pi G m_{p}^{2}\right)=1.17 \times 10^{38} \mathrm{~cm}^{-1} \mathrm{~K}^{-1}$. We note that the scale height in Zacharias et al. (2017) contains a minor calculation error on the order of unity, which has been corrected here. As clouds cannot be infinitely large, we define an outer radius $R$ beyond which the density is set to zero.

As the isothermal cloud predominantly contains hydrogen, the sound speed is simply $c_{\mathrm{s}}=\left(5 k_{\mathrm{B}} T / 3 m_{\mathrm{p}}\right)^{1 / 2}$. Using Eq. (3), this becomes

$$
\begin{aligned}
c_{\mathrm{s}} & =\sqrt{\frac{5 k_{\mathrm{B}} n_{0} r_{0}^{2}}{3 m_{\mathrm{p}} \tilde{c}}} \\
& =1.4 \times 10^{5}\left(\frac{n_{0}}{10^{15} \mathrm{~cm}^{-3}}\right)^{1 / 2}\left(\frac{r_{0}}{4 \times 10^{12} \mathrm{~cm}}\right) \mathrm{cm} \mathrm{s}^{-1},
\end{aligned}
$$

which shows that all relevant speeds are much larger than the cloud's sound speed. This verifies a posteriori that large Mach numbers are achieved and a strong shock is formed in the cloud.

\subsection{Necessary jet condition}

The ablation process commences if the cloud's gravitational pull cannot withstand the jet's ram pressure. While the details of the process require MHD simulations, which are beyond the scope of this paper, we can provide a rough estimate of the necessary jet condition to ablate the cloud.

In the frame of the host galaxy, the relativistic jet containing a fraction of $a$ cold protons and $(1-a)$ positrons per electron, exerts the ram pressure

$P_{R} \approx \Gamma(\Gamma-1) a m_{\mathrm{p}} c^{2} n_{j}$

with the bulk Lorentz factor $\Gamma$, the proton rest energy $m_{\mathrm{p}} c^{2}$, and the jet's electron density $n_{j}$. We assumed that $a m_{\mathrm{p}}>\bar{\gamma} m_{\mathrm{e}}$, where $\bar{\gamma}$ is the average electron Lorentz factor. This inequality implies that the mass of protons is greater than the average relativistic mass of the electrons. In turn, the ram pressure is dominated by the protons. This approximation is improved if the protons have non-negligible kinetic energy in the comoving frame.

The ram pressure must overcome the cloud's pressure on its particles. Following the hydrostatic equilibrium condition, the cloud's pressure is

$P_{\mathrm{c}}(r)=\frac{n_{0} k_{\mathrm{B}} T}{1+\left(r / r_{0}\right)^{2}} \approx n_{0} k_{\mathrm{B}} T$

where the approximation holds within the cloud's center $\left(r \ll r_{0}\right)$.

If the ram pressure is larger than the cloud's central pressure, the cloud is destructed entirely. Setting $P_{R}>P_{\mathrm{c}}\left(r \ll r_{0}\right)$ and solving for the bulk Lorentz factor results in

$$
\begin{aligned}
\Gamma(\Gamma-1) & >\frac{n_{0} k_{\mathrm{B}} T}{a m_{\mathrm{p}} c^{2} n_{j}} \\
& =128\left(\frac{n_{0}}{10^{15} \mathrm{~cm}^{-3}}\right)\left(\frac{T}{140 \mathrm{~K}}\right)\left(\frac{a}{0.1}\right)^{-1}\left(\frac{n_{j}}{10^{3} \mathrm{~cm}^{-3}}\right)^{-1} .
\end{aligned}
$$

The square root provides us with the required value for the bulk Lorentz factor of $\Gamma \gtrsim 12$, which is achieved in many blazar jets (e.g., Jorstad et al. 2017). Hence, a cloud with the provided parameters is indeed destructed. Parts of a denser cloud, or one with a higher temperature, may possibly survive the encounter. We note that the chances of the cloud's survival are significantly reduced if the jet protons are not cold. As a side note, while stars may be stripped of their astrospheres, the star itself should survive the encounter with a relativistic jet.
Additionally, we can consider the time the cloud needs to cross the jet. As the central region of the cloud within the scale height is the densest part of the cloud, we consider this size in the following estimate. The ablation process is governed by the shock that forms during the interaction. Hence, the crossing time of the shock through the cloud is a good estimator of the ablation time since it disrupts the internal structure of the cloud, adding (or enhancing) the turbulent motions in the cloud, which weakens the gravitational pull. We can derive the minimum shock speed $v_{\mathrm{s}}$ required to cross the cloud before the cloud with a speed of $v$ has crossed the jet of radius $R_{j}$ :

$$
\begin{aligned}
t_{\text {cross }}= & \frac{2 R_{j}}{v}<t_{\mathrm{s}}=\frac{2 r_{0}}{v_{\mathrm{s}}} \\
\Leftrightarrow & v_{\mathrm{s}}<\frac{r_{0}}{R_{j}} v \\
= & 3.2 \times 10^{3}\left(\frac{r_{0}}{4 \times 10^{12} \mathrm{~cm}}\right)\left(\frac{v}{2 \times 10^{7} \mathrm{~cm} \mathrm{~s}^{-1}}\right) \\
& \times\left(\frac{R_{j}}{2.5 \times 10^{16} \mathrm{~cm}}\right)^{-1} \mathrm{~cm} \mathrm{~s}^{-1} .
\end{aligned}
$$

If we express the jet radius as a function of the distance $z_{j}$ from the black hole using $R_{j}=z_{j} \tan \Gamma^{-1} \approx z_{j} / \Gamma$, Eq. (8) becomes

$$
\begin{aligned}
v_{\mathrm{s}}< & \frac{r_{0} \Gamma}{z_{j}} v \\
= & 1.3 \times 10^{3}\left(\frac{r_{0}}{4 \times 10^{12} \mathrm{~cm}}\right)\left(\frac{v}{2 \times 10^{7} \mathrm{~cm} \mathrm{~s}^{-1}}\right) \\
& \times\left(\frac{\Gamma}{10}\right)\left(\frac{z_{j}}{6.5 \times 10^{17} \mathrm{~cm}}\right)^{-1} \mathrm{~cm} \mathrm{~s}^{-1}
\end{aligned}
$$

Comparing this to our earlier estimate that the shock speed may actually be on the order of $\sim c / 4$, it appears that the cloud is not able to cross the jet in time. This is ncloud electrons into the emission ot the case for clouds that are close to the base of the jet, where the speed of motion of the cloud is a lot higher and the jet is substantially narrower.

The gradual ablation of the cloud results in the injection of the cloud particles into the jet, where they get mixed into the bulk flow. The injection function is derived in the next section.

\subsection{Injection function into the jet emission region}

The number of particles $\mathrm{d} N$ entering the jet in a given time step $\mathrm{d} t$ depends on the density of the cloud and the ablated volume $\mathrm{d} V$ of a slice of the cloud. As in Zacharias et al. (2017), with $x=0$ we denote the point of the cloud that first touches the jet; $x=R$ is the center of the cloud and $x=2 R$ is the far side of the cloud. The ablated volume at position $x$ becomes (Zacharias \& Schlickeiser 2013)

$\mathrm{d} V(x)=\mathrm{d} x \int^{x} \mathrm{~d} A(\tilde{x})=\pi\left(2 R x-x^{2}\right) \mathrm{d} x$,

with the width $\mathrm{d} x$ and the cross-section $A(x)$ of the ablated volume. The particle number in a slice yields

$$
\begin{aligned}
\mathrm{d} N(x) & =\mathrm{d} x \int^{x} n(r) \mathrm{d} A(\tilde{x}) \\
& =\pi n_{0} \mathrm{~d} x r_{0}^{2} \ln \left(\frac{r_{0}^{2}+R^{2}}{r_{0}^{2}+(R-x)^{2}}\right) .
\end{aligned}
$$

If the cloud enters the jet with a constant speed $v$, the length scales can be transformed to time scales. Then the number of 
particles entering the jet in a given time step $\mathrm{d} t=\mathrm{d} x / v$ is

$$
\frac{\mathrm{d} N(t)}{\mathrm{d} t}=\pi n_{0} v r_{0}^{2} \ln \left(\frac{t_{0}^{2}+t_{R}^{2}}{t_{0}^{2}+\left(t_{R}-t\right)^{2}}\right),
$$

with $t_{0}=r_{0} / v$ and $t_{R}=R / v$.

In the simulations below, the radiation is calculated in the comoving frame of the jet. Hence, the particle rate, Eq. (12), must be transformed to the comoving frame of the jet ${ }^{3}$. The jet flows with the bulk Lorentz factor $\Gamma$, and we assume that the cloud enters the jet in a right angle in the galactic frame. The Lorentz transformation of the time step is $\mathrm{d} t=\Gamma \mathrm{d} t^{\prime}$, while the transformation of the time coordinate is $t=t^{\prime} / \Gamma$ due to the right angle. Then, in the comoving frame, the particle rate becomes:

$$
\frac{\mathrm{d} N\left(t^{\prime}\right)}{\mathrm{d} t^{\prime}}=\Gamma \pi n_{0} v r_{0}^{2} \ln \left(\frac{\left(\Gamma t_{0}\right)^{2}+\left(\Gamma t_{R}\right)^{2}}{\left(\Gamma t_{0}\right)^{2}+\left(\Gamma t_{R}-t^{\prime}\right)^{2}}\right) .
$$

These initially thermal particles accelerate in the jet through a process which we do not specify here, and they are subsequently injected into the emission region (for more in-depth models, see e.g., Chen et al. 2012; Weidinger \& Spanier 2015; Böttcher \& Baring 2019). We assume that a fraction $\epsilon_{c} \sim 0.1$ (e.g., Sironi et al. 2013) of the cloud electrons is accelerated and the resulting spectrum is a power-law with the index $s^{\prime}$ between a minimum and maximum Lorentz factor of $\gamma_{\min ^{\prime}}$ and $\gamma_{\text {max }^{\prime}}$, respectively. We stress again that we assume that the hadronic cloud particles remain energetically cold. The injection luminosity of cloud electrons into the emission region of the jet becomes

$$
\begin{aligned}
L_{\text {inj,c }}^{\prime}(t) & =m_{\mathrm{e}} c^{2} \epsilon_{\mathrm{c}} \frac{\mathrm{d} N\left(t^{\prime}\right)}{\mathrm{d} t^{\prime}} \int_{\gamma_{\min }^{\prime}}^{\gamma_{\max }^{\prime}} \gamma^{\prime 1-s^{\prime}} \mathrm{d} \gamma^{\prime} \\
& =m_{\mathrm{e}} c^{2} \epsilon_{\mathrm{c}} \frac{\mathrm{d} N\left(t^{\prime}\right)}{\mathrm{d} t^{\prime}} \begin{cases}\frac{\ln \left(\gamma_{\max }^{\prime} / \gamma_{\min }^{\prime}\right)}{\gamma_{\min }^{\prime-1}-\gamma_{\max }^{\prime-1}} & s^{\prime}=2 \\
\frac{1}{2-s^{\prime}}\left(\gamma_{\max }^{\prime 2-s^{\prime}}-\gamma_{\min }^{\prime 2-s^{\prime}}\right) & \text { else. }\end{cases}
\end{aligned}
$$

While the time dependency in Eq. (14) is obviously the same as in Zacharias et al. (2017), here we have also derived the full transformation and the correct normalization factor. These were only indirectly considered or treated as free parameters in Zacharias et al. (2017). Therefore, within the given assumptions, Eq. (14) provides the correct particle injection function of a slowly ablated cloud into the emission region of a jet.

\section{Parameter study}

In this section we provide a thorough study on the resulting lightcurves following a scan over the parameters of the cloud and the emission region. Here and in the following, we use the time-dependent, leptonic one-zone code, which is extensively described in Diltz \& Böttcher (2014) and Zacharias et al. (2017). The emission region parameters are given and defined in Table 1, providing cases for both flat spectrum radio quasars (FSRQs) and BL Lac objects. To study FSRQs, we used the same parameters as Zacharias et al. (2017). The emission processes that are included are synchrotron, synchrotron-self Compton (SSC), and inverse-Compton scattering of photons from the accretion disk and the BLR. The parameters provided in Table 1 imply an initial density of nonthermal electrons of $8 \times 10^{3} \mathrm{~cm}^{-3}$ for FSRQs

3 Quantities in the comoving frame are denoted with primes.
Table 1. Jet emission region parameter definition, symbol and value for the FSRQ, and BL Lac object cases.

\begin{tabular}{lccc}
\hline \hline Definition & Symbol & FSRQ & BL Lac object \\
\hline Distance to black hole & $z$ & $6.5 \times 10^{17} \mathrm{~cm}$ & $1.0 \times 10^{19} \mathrm{~cm}$ \\
Doppler factor & $\delta$ & 35 & 35 \\
Emission region radius & $R_{j^{\prime}}$ & $2.5 \times 10^{16} \mathrm{~cm}$ & $1.0 \times 10^{17} \mathrm{~cm}$ \\
Magnetic field strength & $B_{j^{\prime}}$ & $3.7 \mathrm{G}$ & $1.0 \mathrm{G}$ \\
$\mathrm{e}^{-}$injection luminosity & $L_{\text {inj }}$ & $2.2 \times 10^{43} \mathrm{erg} \mathrm{s}^{-1}$ & $5.0 \times 10^{42} \mathrm{erg} \mathrm{s}^{-1}$ \\
Min. $\mathrm{e}^{-}$Lorentz factor & $\gamma_{\min }^{\prime}$ & $1.3 \times 10^{1}$ & $1.6 \times 10^{2}$ \\
Max. $\mathrm{e}^{-}$Lorentz factor & $\gamma_{\max }^{\prime}$ & $3.0 \times 10^{3}$ & $3.0 \times 10^{6}$ \\
$\mathrm{e}^{-}$spectral index & $s^{\prime}$ & 2.4 & 2.2 \\
Escape time scaling & $\eta_{\mathrm{esc}}^{\prime}$ & 10.0 & 10.0 \\
BLR Temperature & $T_{\mathrm{BLR}}$ & $5.0 \times 10^{4} \mathrm{~K}$ & - \\
Cosmological redshift & $z_{\text {red }}$ & 1.037 & 1.037 \\
\hline
\end{tabular}

Table 2. Baseline cloud parameter definition, symbol, and value for the artificial clouds.

\begin{tabular}{lcc}
\hline \hline Definition & Symbol & Value \\
\hline Cloud radius & $R$ & $6.0 \times 10^{13} \mathrm{~cm}$ \\
Cloud scale height & $r_{0}$ & $4.0 \times 10^{12} \mathrm{~cm}$ \\
Cloud density & $n_{0}$ & $1.0 \times 10^{15} \mathrm{~cm}^{-3}$ \\
Cloud speed & $v$ & $2.0 \times 10^{7} \mathrm{~cm} \mathrm{~s}^{-1}$ \\
Acceleration efficiency & $\epsilon_{\mathrm{c}}$ & 0.1 \\
\hline
\end{tabular}

and $7 \times 10^{2} \mathrm{~cm}^{-3}$ for BL Lac objects, respectively ${ }^{4}$. We note that the redshift in Table 1 is required for the flux normalization.

The baseline cloud parameters from which we conduct the parameter study of this section are given in Table 2. It is important to note that the injection luminosity provided by Eq. (14) was added on top of the jet values listed in Table 1. We assume that the electrons supplied from the ablation process are accelerated to the same spectral behavior, namely to the same minimum and maximum Lorentz factors and spectral index, as the already existing jet electrons. This is reasonable if they are accelerated in the same environment at a shock downstream of the jet-cloud interaction site.

For the parameter study, each cloud parameter was varied individually, resulting in the four plots shown in Fig. 2, which display the lightcurves in the $\gamma$-ray-, X-ray-, and $R$-band utilizing the FSRQ jet parameters. The results are as follows.

(a) In regards to the variation of cloud radius $R$, as expected, the flare takes longer to evolve with a larger $R$, while the peak flux also increases mildly. A larger radius overall implies a larger number of cloud particles, thus explaining the mild increase in flux.

(b) With respect to the variation of scale height $r_{0}$, as $r_{0}$ governs the size of the region with a constant, maximal density $n_{0}$, the total number of particles significantly changes with a variation of $r_{0}$. For a small $r_{0}$, the number of particles in the cloud becomes so low that a variation in flux is barely visible (magenta curve). On the other hand, a larger $r_{0}$ not just increases the flux, but it also changes the curvature of the lightcurve, owing to the combination of higher particle numbers in a larger cloud volume.

\footnotetext{
4 We note that the seemingly high density in the FSRQ case is in accordance with the modeling of CTA 102 by Zacharias et al. (2017), while the BL Lac case is an adaptation from the FSRQ values. Both values are comparable to what was found in other studies (e.g., Böttcher et al. 2013; Zacharias \& Wagner 2016).
} 

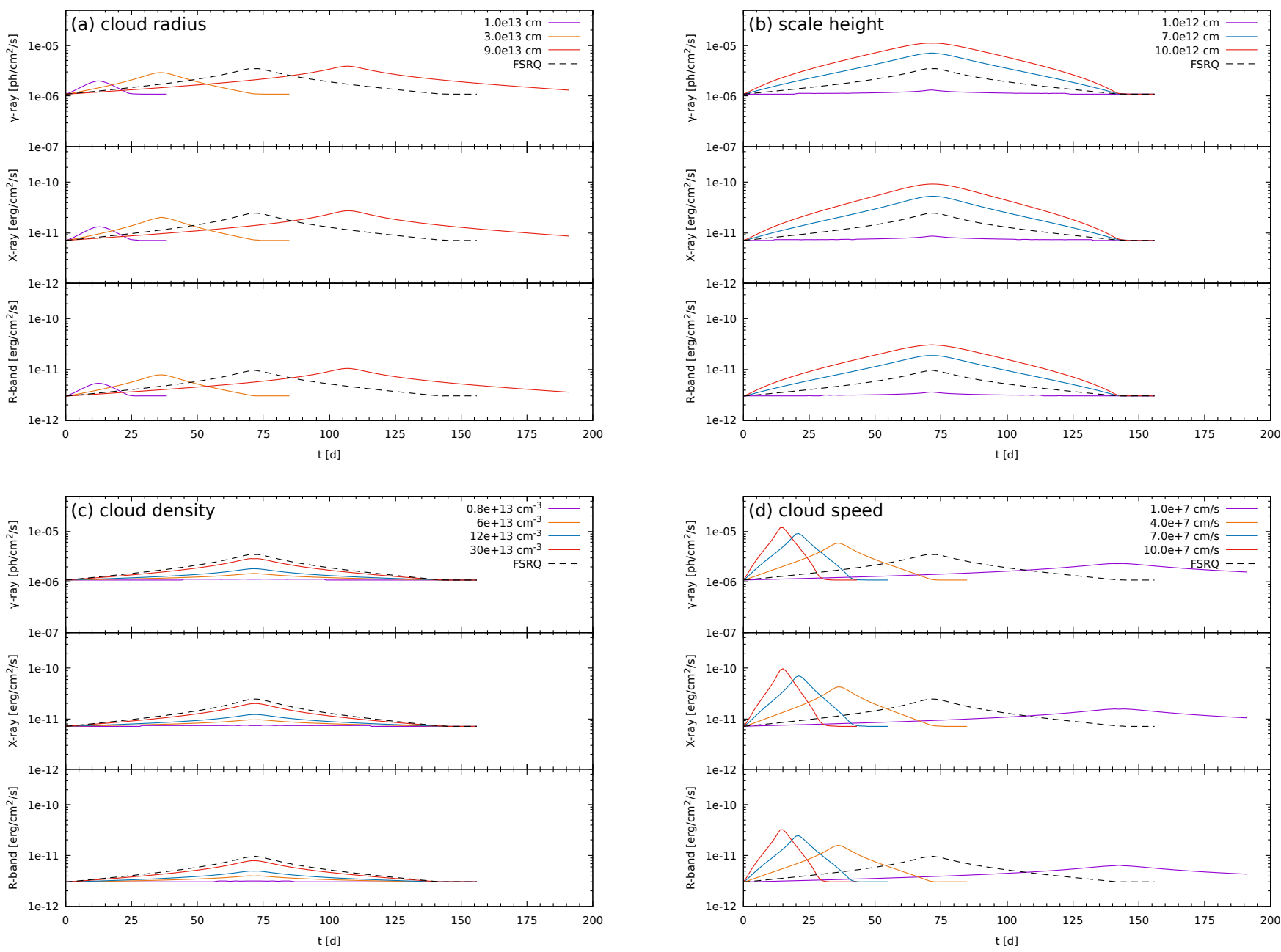

Fig. 2. Lightcurves in the observer's frame for different parameter values of theoretical clouds. In each panel, lightcurves in the $\gamma$-ray, X-ray, and $R$ band are shown for the following different, varied parameters: $(a)$ cloud radius $R,(b)$ scale height $r_{0},(c)$ cloud density $n_{0}$, and $(d)$ cloud speed $v$. The black-dashed line represents the lightcurve employing the baseline parameters given in Table 2. We note the logarithmic $y$-axes.

In turn, the peak becomes less pronounced with increasing scale height.

(c) In the case of the variation of density $n_{0}$, the peak fluxes are linearly altered as the synchrotron and external-Compton ${ }^{5}$ processes linearly depend on the particle density.

(d) Regarding the variation of speed $v$, the influence of $v$ on the lightcurves is involved as it changes both the normalization factor and the duration of the event. Therefore, slower speeds result in longer, but less pronounced flares, which is in line with the discussion in Sect. 2.

Obviously, the cloud parameters have a strong influence on the lightcurves, resulting in a zoo of potential solutions. This could explain many symmetrical flares.

However, the parameters of the emission region itself may also influence the lightcurve. We have tested this by individually varying the magnetic field $B_{j}^{\prime}$ and the size $R_{j}^{\prime}$ of the emission region as well as the spectral index $s^{\prime}$ of the electron distribution and the jet injection luminosity $L_{\text {inj }}^{\prime}$. The baseline cloud parameters are unchanged. The results are shown in Fig. 3 and the details are as follows.

(a) With respect to the variation of the magnetic field $B_{j}^{\prime}$, the synchrotron component (optical band) obviously directly reacts to changes in the magnetic field, while the $\gamma$-ray component

\footnotetext{
5 SSC is negligible in this parameter set.
}

(external Compton on BLR in this case) remains at the same flux level for most cases and starts to decrease for high magnetic field strengths. This decrease is expected as the synchrotron cooling begins to dominate the external-Compton cooling, resulting in a decreased efficiency in the external-Compton process. Similar statements can be made for the X-ray domain that exhibit similar fluxes for most magnetic field values and only deviate for the highest magnetic field strengths. Here, the SSC process starts to dominate the external-Compton process in this energy range.

(b) With regard to the variation of the size $R_{j}^{\prime}$, there is no noteworthy change in the lightcurves for most values. At the smallest size, the SSC process dominates in the X-ray domain due to the increased densities in the synchrotron photons. At the largest size, the dynamical and escape timescales become so long, in comparison to the chosen time step of $1 \mathrm{~d}$ in the observer's frame, that particles remain much longer in the emission region and the fluxes decrease at a slower rate than they rise.

(c) Pertaining to the variation of the electron spectral index $s^{\prime}$, following Eq. (14), the shape of the accelerated (i.e., injected) particle distribution has a strong influence on the injection luminosity of the cloud particles. While the total number of particles does not change, their influence is distributed to higher energies for harder electron distributions. In turn, the variation is more pronounced. The opposite is true for softer electron distributions. 

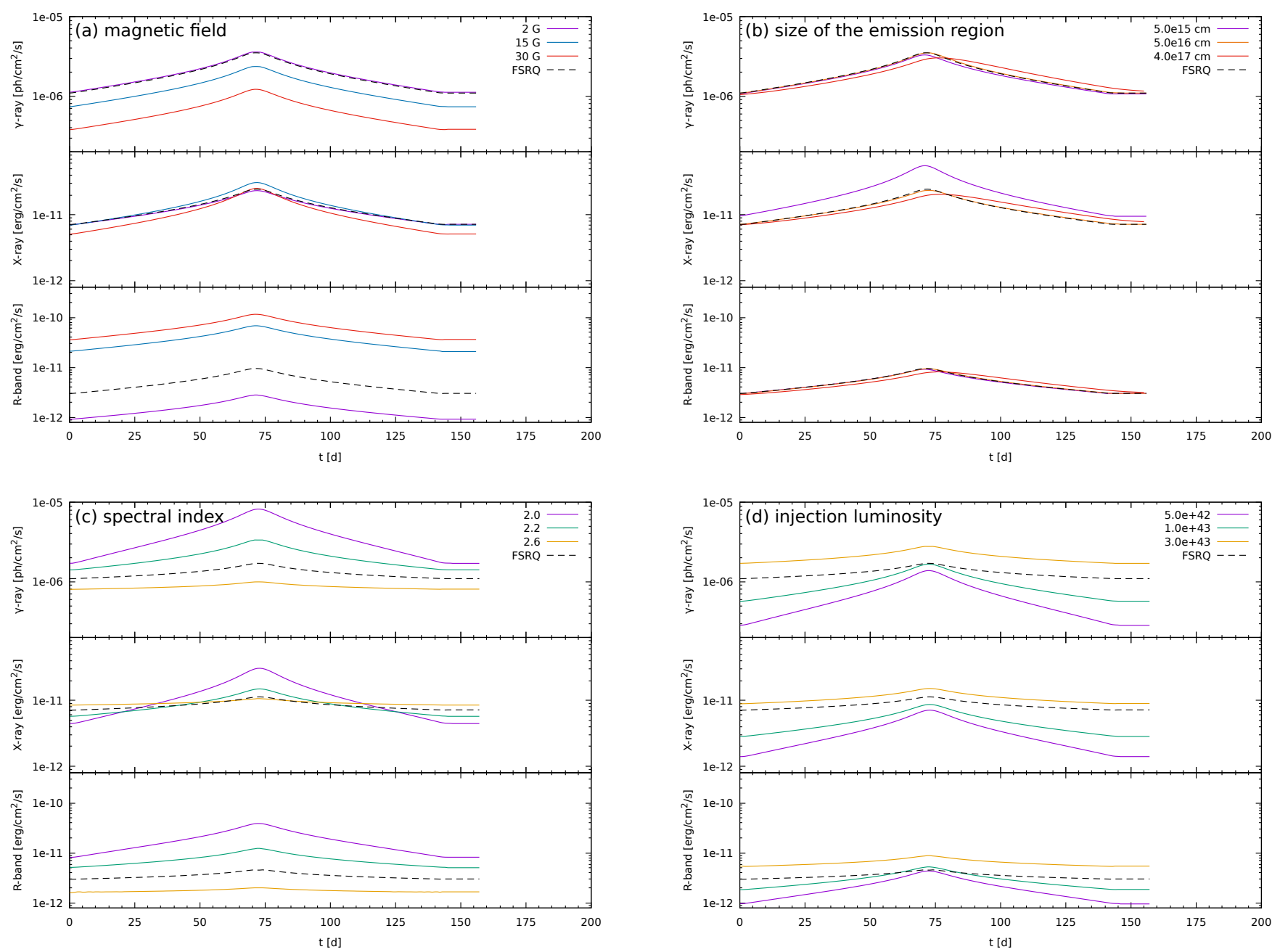

Fig. 3. Lightcurves in the observer's frame of theoretical clouds for different parameter values of the jet emission region. In each panel, lightcurves in the $\gamma$-ray, X-ray, and $R$ band are shown for the following different varied parameters: $(a)$ magnetic field $B_{j}^{\prime}$, and $(b)$ size $R_{j}^{\prime}$ of the emission region, as well as $(c)$ spectral index $s^{\prime}$ of the electron distribution, and $(d)$ the injection luminosity $L_{\text {inj }}^{\prime}$ of the quiescent state. The black-dashed line marks the lightcurve employing the FSRQ parameters given in Table 1. We note the logarithmic $y$-axes.

(d) As to variation of the injection luminosity $L_{\text {inj }}^{\prime}$, this is the injection luminosity of the initial emission region, and its value plays a significant role in the observed variability. For larger values, the luminosity added by the cloud is relatively small and the variation does not emerge strongly from the quiescence state. On the other hand, for a small injection luminosity, the cloud injection becomes significant and displays more pronounced variability.

As a final test, we have considered a typical BL Lac object parameter set (e.g., Böttcher et al. 2013) with parameters provided in Table 1. The lightcurve is shown in Fig. 4. The changes in the parameters of the emission region compared to the FSRQ case concern a larger distance from the black hole in order to avoid inverse-Compton scattering on external photon fields, which are located at smaller distances (Zacharias et al. 2019) and have a larger emission region, a smaller magnetic field, a smaller injection luminosity, and a harder and more energetic electron distribution. Following the discussion on individual changes of emission region parameters, we can expect a significant change in the lightcurve behavior.

Indeed, the lightcurves in the BL Lac object case considerably differ from the FSRQ case, which is shown as the blackdashed line in Fig. 4, despite using the same cloud parameters.
The variation in all energy bands exceeds one order of magnitude and even two orders of magnitude in the $\gamma$-ray band. The latter can easily be understood since the SSC process depends on the particle distribution quadratically. Hence, as the only change in the cloud injection is the particle number, the SSC flux must change quadratically compared to the synchrotron component. The stronger reaction in the X-ray domain is amplified compared to the FSRQ case since this energy band is now produced by highly energetic electrons emitting synchrotron emission, which is much more variable than in the baseline case where the X-ray band is dominated by low-energetic electrons producing inverseCompton emission.

\section{Interstellar objects}

Before we proceed, a note is required on the free parameters. As we have used the scale height as a free parameter in the previous section, we implicitly considered the cloud temperature $T$ to be a dependent variable. However, unlike the scale height, $T$ is a measurable quantity and, therefore, the scale height becomes the dependent variable from now on. Interestingly, the injection rate, Eq. (13), is proportional to $n_{0} r_{0}^{2}$. By inserting Eq. (3), this becomes $n_{0} r_{0}^{2}=\tilde{c} T$, independent of the density. Hence, the 


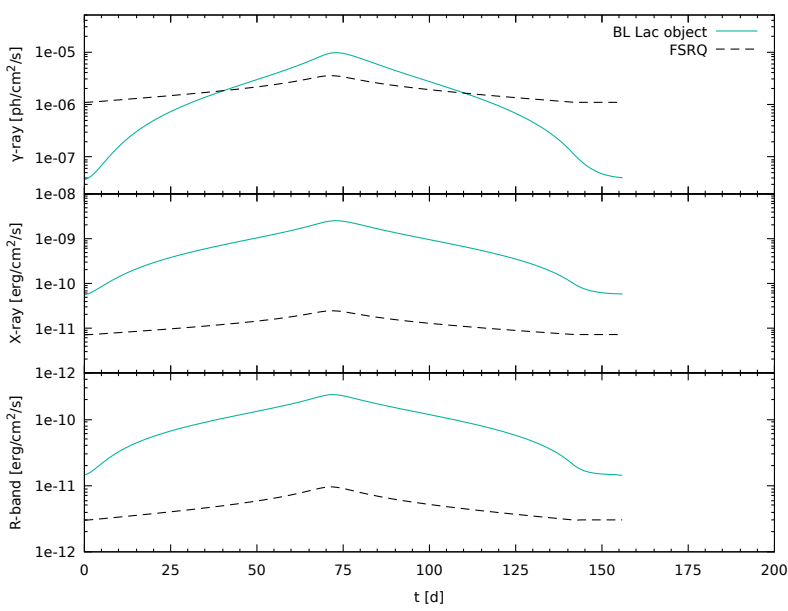

Fig. 4. Lightcurve in the observer's frame of a theoretical cloud employing the BL Lac object parameter set (turquoise line) of the emission region compared to the FSRQ parameter set (black-dashed line). The parameter sets are given in Table 1. We note the logarithmic $y$-axes.

influence of the density on the lightcurves is minor. The injection is therefore driven by the speed and the temperature of the cloud.

With this in mind, we can discuss the lightcurves from interstellar clouds. Some cloud types and their typical parameters (Carroll \& Ostlie 2007) are given in Table 3. Clearly, this list is not exhaustive, and it should be considered an example. All of these clouds fulfill the ablation conditions in Eqs. (7) and (8).

As we have seen in the previous section, the speed of the cloud has an enormous influence on the resulting lightcurve. Hence, stronger flares may be expected for clouds that are relatively close to the black hole. Given that many AGNs are located in elliptical hosts, the nuclear activity may be a result of the merger of galaxies. In the process, gas and dust (in the form of clouds) are pushed into the galactic center. In turn, many clouds come close to the black hole and the jet while moving rapidly. This provides the necessary ingredients for our model. We assume that the clouds have reached a distance to the black hole within the radius of the BLR and hence move with roughly the orbital speed of the BLR - about $5000 \mathrm{~km} \mathrm{~s}^{-1}$. This allows us to use the FSRQ model. Additionally, as the BL Lac object scenario amplifies the variability, we also provide the corresponding lightcurves.

The resulting lightcurves are shown in Fig. 5 with dashed lines for the FSRQ parameters and solid lines for the BL Lac object model. Obviously, all cases exhibit strong flares, which vary in duration and peak flux. The evolution of the lightcurve also changes slightly in accordance with the discussion from the previous section. The duration is, of course, governed by the size of the objects - and they are ordered in decreasing size - so the duration drops from case to case. The peak flux in turn depends on the particle number, which depends on both the size and the density. For the FSRQ parameter set, the flux variation is on the order of a few in all three bands. In the BL Lac object case, the statements of the previous section hold that the variation in the $\gamma$-ray band is roughly quadratically the variation in the X-ray and $R$ bands. That is, if variations in the $\mathrm{X}$-ray and $\mathrm{R}$ bands are on the order of one order of magnitude, the $\gamma$-ray band exhibits variations on the order of two orders of magnitude. The most notable variation takes place in "hot cores", which is the densest and hottest of the examples. In this case, the lightcurve varies more than an order of magnitude even in the FSRQ case.
Astrospheres of RGB stars are another common "cloud" type in elliptical galaxies. We discuss their case in the appendix as they are not fully compatible with our assumptions on the derivation of the cloud's density structure.

\section{Discussion}

Elliptical galaxies - the hosts of blazars - form through the collision of gas-rich spiral galaxies. A lot of the free gas is funneled into the center of the galaxy, where an AGN is turned on. Clouds of gas enter the galactic center from any direction, and they may encounter the relativistic jet. We have considered this type of an encounter of a cloud with the jet as a particle injection process that produces long-lasting flares.

We first derived the analytical equations that describe the injection function of a spherical, isothermal gas cloud that is only shaped by the hydrostatic equilibrium between its selfgravity and its gas pressure. This expanded upon the work of Zacharias et al. (2017) and provides the correct normalization of the injection process. We also derived the necessary jet conditions to fully ablate the cloud. It turns out that jets should be able to ablate most cloud types. We then proceeded to study the theoretical lightcurve shapes by considering various parameter sets for both the cloud and the jet. The most important cloud parameter in this regard is the scale height, which depends on the temperature and the central density of the cloud. The scale height's value relative to the cloud radius determines the homogeneity of the cloud. Homogeneous clouds, that is to say those with a large scale height, produce round lightcurves with a steep rise and decay as well as a relatively flat maximum, while clouds with a small scale height produce a peaked lightcurve with a flat rise and decay as well as a pronounced central peak. As the density of the cloud can be determined from the peak flux of the lightcurve, the shape of the lightcurve gives a strong indication of the temperature of the cloud.

While the jet parameters can be deduced from observations before the flare, they also have a significant influence on the lightcurve. Most notably, parameters describing BL Lac objects produce a significantly larger variability than FSRQ parameters. While this changes the lightcurve shape, it has no major influence on the peak-structure and, therefore, on the possibility of determining the scale height. This is important as it does not influence the predictive power of the model.

Subsequently, we used examples of different cloud types that may be present in an active galaxy and may penetrate the jet. Each example results in variable fluxes with different magnitudes, different lightcurve shapes, and variations on different timescales. For the example clouds, the variations take thousands to millions of days, which is obviously too long for proper observations. However, much smaller clouds are present in the Universe, such as the very dense structures around forming stars. Furthermore, the peaks in Fig. 5 are quite pronounced in terms of flux variation and duration, lasting only for 1 or $2 \%$ of the entire high state duration. This increases the observational potential.

One of our major assumptions is that all clouds abide by the same hydrostatic density structure. While this is a relatively simple structure, real clouds are much more complex. Especially in star forming regions, the cloud structure is chaotic (Keto et al. 2020; Xu \& Lazarian 2020) and shaped from gravitational encounters, stellar winds, magnetic fields, etc. It is rather likely that these types of nonspherically-symmetric structures produce flares that are asymmetric. Furthermore, one can also expect a more complicated density structure with several cores and a turbulent behavior. Additionally, we have treated each 
Table 3. Parameters of interstellar clouds.

\begin{tabular}{lccccccc}
\hline \hline & Type & $\begin{array}{c}R \\
{[\mathrm{~cm}]}\end{array}$ & $\begin{array}{c}T \\
{[\mathrm{~K}]}\end{array}$ & $\begin{array}{c}n_{0} \\
{\left[\mathrm{~cm}^{-3}\right]}\end{array}$ & $\begin{array}{c}v \\
{\left[\mathrm{~cm} \mathrm{~s}^{-1}\right]}\end{array}$ & $\begin{array}{c}r_{0} \\
{[\mathrm{~cm}]}\end{array}$ & Ablate? \\
\hline (a) & Giant molecular clouds & $7.7 \times 10^{19}$ & 15 & $2.0 \times 10^{8}$ & $5.0 \times 10^{8}$ & $3.0 \times 10^{15}$ & Yes \\
(b) & Dark clouds & $1.5 \times 10^{19}$ & 10 & $5.0 \times 10^{8}$ & $5.0 \times 10^{8}$ & $1.5 \times 10^{15}$ & Yes \\
(c) & Clumps & $1.0 \times 10^{19}$ & 10 & $1.0 \times 10^{9}$ & $5.0 \times 10^{8}$ & $1.1 \times 10^{15}$ & Yes \\
(d) & Bok globules & $1.2 \times 10^{18}$ & 10 & $4.0 \times 10^{10}$ & $5.0 \times 10^{8}$ & $1.7 \times 10^{14}$ & Yes \\
(e) & Dense cores & $1.5 \times 10^{17}$ & 10 & $1.0 \times 10^{10}$ & $5.0 \times 10^{8}$ & $3.4 \times 10^{14}$ & Yes \\
(f) & Hot cores & $1.0 \times 10^{17}$ & 200 & $1.0 \times 10^{14}$ & $5.0 \times 10^{8}$ & $1.5 \times 10^{13}$ & Yes \\
\hline
\end{tabular}

Notes. The scale height $r_{0}$ was calculated from the other parameters and not a free parameter. $R$ - cloud radius; $T$ - cloud temperature; $n_{0}-$ cloud density; $v$-cloud speed; $r_{0}$ - derived cloud scale height; Ablate? - If the cloud and jet parameters (Table 1) fulfill Eqs. (7) and (8).
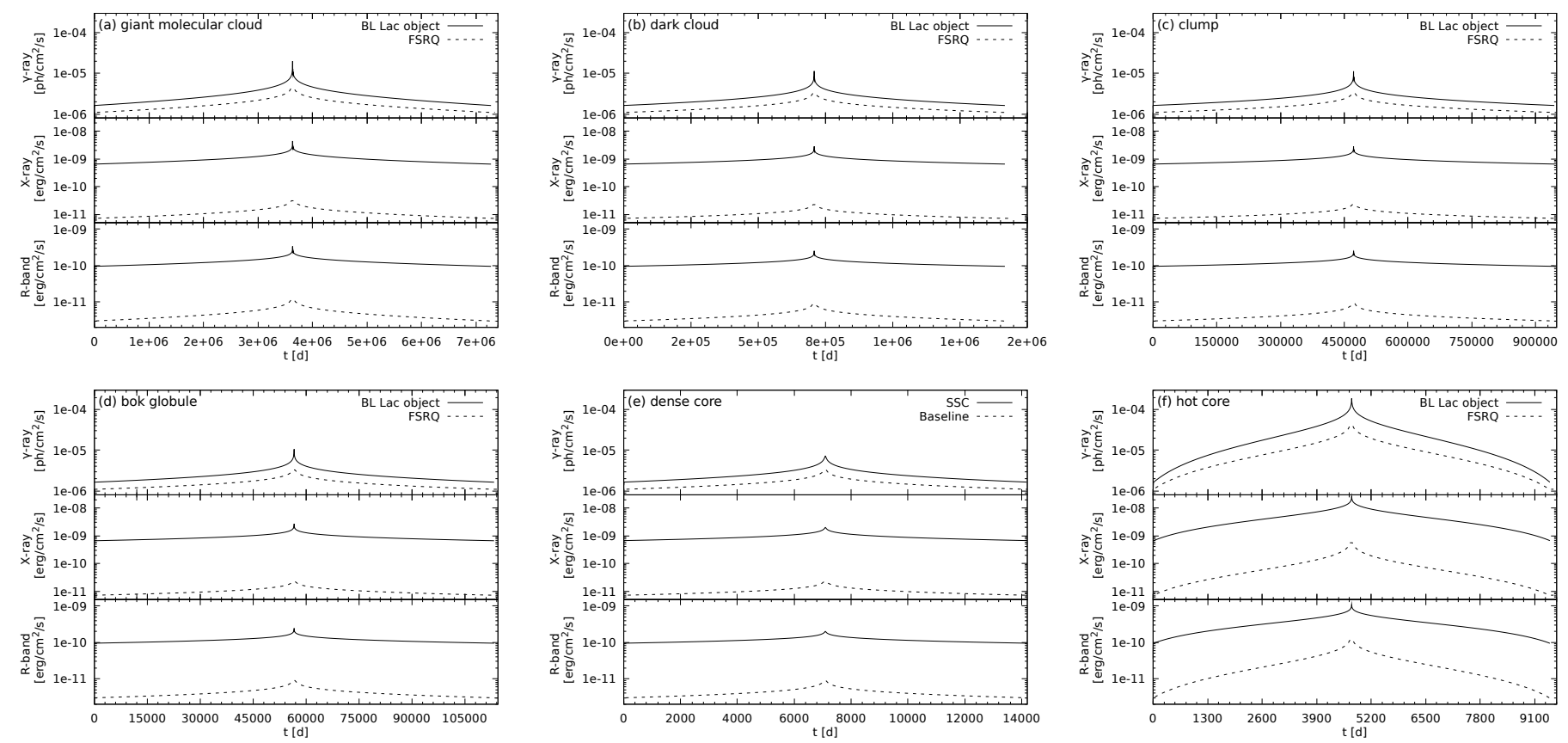

Fig. 5. Lightcurves of the FSRQ (dashed line) and BL Lac object (solid line) cases in the observer's frame for three energy bands for examples of real clouds: $(a)$ giant molecular cloud, $(b)$ dark cloud, $(c)$ clump, $(d)$ bok globule, $(e)$ dense core, and $(f)$ hot core. The jet parameters are given in Table 1 and the cloud parameters are provided in Table 3 . We note the logarithmic $y$-axes.

cloud as an individual entity. In fact, many of the cloud types considered are part of star-forming regions, and they are intertwined. This type of multi-cloud model might produce bright flares on top of an extended high state. These are intriguing possibilities for further applications of the model. In any case, the lightcurve would become more complicated with several peaks. A similar result would be obtained if several individual clouds were to interact with the jet at the same time (e.g., del Palacio et al. 2019). While this increases the number of ablated particles and, thus, the flux variation, one would observe several peaks again. Disentangling the different clouds may be a complicated endeavor.

In our simulations, we have assumed that every particle of the cloud enters the jet. This is unlikely to happen. The interaction of the loosely bound cloud with the highly energetic jet results in the ejection of cloud material, and only a fraction of the cloud enters the jet. It is difficult to quantify how much material is lost in this way, and it would require dedicated MHD simulations, which are beyond the scope of this paper. Additionally, in the case of real clouds, it is unlikely that all particles enter the jet independently of the cloud size with respect to the jet size.
If the cloud is much larger than the jet, parts of the cloud would skip the jet. However, if the central and densest part of the cloud enters the jet, the effect should be minor. In fact, in all of our examples, the scale height is smaller than the jet radius. Despite these issues, a significant number of particles enter the jet, thus producing an equally significant flux variation.

We have considered leptonic synchrotron and inverseCompton radiation processes since these constitute the standard blazar emission scenario. However, as the cloud naturally contains protons and heavier nuclei, hadronic radiation processes might be an interesting alternative to produce the emission. On the one hand, the cloud's nuclei may be accelerated to nonthermal speeds and produce radiation on their own (proton synchrotron, pion and muon synchrotron, etc.; e.g., Böttcher et al. 2013; Zacharias et al. 2019). On the other hand, the cloud's nuclei could also serve as targets for the jet's relativistic protons, resulting in proton-nucleus interactions and subsequent radiation production (Hörbe et al. 2020). This is an intriguing possibility to produce a flare without the need to accelerate the cloud particles. Here, as well, dedicated simulations may provide further insights. 
In summary, we have demonstrated that the cloud ablation process is a viable option to produce long-lasting high states in blazars. Further studies, especially MHD or particle-in-cell simulations of the entire process, are strongly encouraged.

Acknowledgements. The authors wish to thank Markus Böttcher, Patrick Kilian, Klaus Scherer, Valentí Bosch-Ramon, Maxim Barkov, Frank Rieger, and Kerstin Weiß for stimulating discussions on model and manuscript details as well as cloud parameters. We also thank the anonymous referee for valuable suggestions that helped to improve the manuscript. Funding by the German Ministry for Education and Research (BMBF) through grant 05A17PC3 is gratefully acknowledged.

\section{References}

Araudo, A. T., Bosch-Ramon, V., \& Romero, G. E. 2010, A\&A, 522, A97 Araudo, A. T., Bosch-Ramon, V., \& Romero, G. E. 2013, MNRAS, 436, 3626 Banda-Barragán, W. E., Federrath, C., Crocker, R. M., \& Bicknell, G. V. 2018, MNRAS, 473, 3454

Barkov, M. V., Aharonian, F. A., Bogovalov, S. V., Kelner, S. R., \& Khangulyan, D. 2012a, ApJ, 749, 119

Barkov, M. V., Bosch-Ramon, V., \& Aharonian, F. A. 2012b, ApJ, 755, 170

Bosch-Ramon, V. 2012, A\&A, 542, A125

Böttcher, M. 2007, Ap\&SS, 309, 95

Böttcher, M., \& Baring, M. G. 2019, ApJ, 887, 133

Böttcher, M., Reimer, A., Sweeney, K., \& Prakash, A. 2013, ApJ, 768, 54

Carroll, B. W., \& Ostlie, D. A. 2007, An Introduction to Modern Astrophysics, 2nd ed. (Cambridge: Cambridge Univ. Press)

Cerruti, M., Zech, A., Boisson, C., \& Inoue, S. 2015, MNRAS, 448, 910

Cerruti, M., Benbow, W., Chen, X., Dumm, J. P., Fortson, L. F., \& Shahinyan, K. 2017, A\&A, 606, A68

Chen, X., Fossati, G., Böttcher, M., \& Liang, E. 2012, MNRAS, 424, 789

Diltz, C., \& Böttcher, M. 2014, JHEAp, 1, 63
Ghisellini, G., Tagliaferri, G., Foschini, L., Ghirlanda, G., et al. 2011, MNRAS, 411, 901

H.E.S.S. Collaboration (Abdalla, H., et al.) 2019, A\&A, 627, A159

Hörbe, M. R., Morris, P. J., Cotter, G., \& Becker Tjus, J. 2020, MNRAS, 496, 2885

Jorstad, S. G., Marscher, A. P., Morozova, D. A., et al. 2017, ApJ, 846, 98

Keto, E., Field, G. B., \& Blackman, E. G. 2020, MNRAS, 492, 5870

Marscher, A. P., \& Gear, W. K. 1985, ApJ, 298, 114

Marscher, A. P., Jorstad, S. G., D’Arcangelo, F. D., et al. 2008, Nature, 452, 966

Origlia, L., Rood, R. T., Fabbri, S., Ferraro, F. R., Fusi, Pecci F., \& Rich, R. M. 2007, ApJ, 667, L85

del Palacio, S., Bosch-Ramon, V., \& Romero, G. E. 2019, A\&A, 623, A101

Parker, E. N. 1958, ApJ, 128, 664

Perucho, M., Bosch-Ramon, V., \& Barkov, M. V. 2017, A\&A, 606, A40

Robinson, R. D., Carpenter, K. G., \& Brown, A. 1998, ApJ, 503, 396

Scherer, K., Baalmann, L. R., Fichtner, H., et al. 2020, MNRAS, 493 , 4172

Schröder, K.-P., \& Cuntz, M. 2005, ApJ, 630, L73

Sironi, L., Spitkovsky, A., \& Arons, J. 2013, ApJ, 771, 54

Torres-Albà, N., \& Bosch-Ramon, V. 2019, A\&A, 623, A91

Urry, C. M., \& Padovani, P. 1995, PASP, 107, 803

Vieyro, F. L., Torres-Alba, N., \& Bosch-Ramon, V. 2017, A\&A, 604, A57

Villaver, E., \& Livio, M. 2009, ApJ, 705, L81

Weidinger, M., \& Spanier, F. 2015, A\&A, 573, A7

Wilkin, F. P. 2000, ApJ, 532, 400

Xu, S., \& Lazarian, A. 2020, ApJ, 890, 157

Zacharias, M. 2018, PoS, 338, 33

Zacharias, M., \& Schlickeiser, R. 2013, ApJ, 777, 109

Zacharias, M., \& Wagner, S. J. 2016, A\&A, 588, A110

Zacharias, M., Böttcher, M., Jankowsky, F., Lenain, J.-P., Wagner, S., \& Wierzcholska, A. 2017, ApJ, 851, 72

Zacharias, M., Böttcher, M., Jankowsky, F., Lenain, J.-P., Wagner, S., \& Wierzcholska, A. 2019, ApJ, 871, 19

Zdziarski, A. A., \& Böttcher, M. 2015, MNRAS, 450, L21 


\section{Appendix A: The astrosphere of RGB stars}

The astrosphere of RGB stars is a special case in our study since they are blown up by the stellar wind, that is, an outward stream of particles. In turn, our assumption of an isothermal cloud is a poor representation of this type of object. Additionally, the gravitational pull that keeps the astrosphere together is provided by the star and not the self-gravity of the cloud. This means that the scale height does not follow from the considerations in Sect. 2, and in fact it loses its meaning. However, we can use the known density structure of astrospheres, which is similar to the hydrostatic case. From some distance $r_{\mathrm{s}, 0}$ above the star's surface up to the termination shock, the density follows an inverse-square law (e.g., Scherer et al. 2020). Following our deliberations in Sect. 2, we assume that the star remains intact during the interaction with the jet. Hence, we can replace the scale height with $r_{\mathrm{s}, 0}$ and our formalism can be applied.

Known parameters of RGB star winds are the mass loss rate $\dot{M}$ (Schröder \& Cuntz 2005; Origlia et al. 2007), the wind velocity $v_{\mathrm{w}}$ (e.g., Robinson et al. 1998), and the radius of the star $R_{\mathrm{S}}$ (e.g., Villaver \& Livio 2009). With these parameters, we can calculate the central density as

$$
\begin{aligned}
n_{0} & =\frac{\dot{M}}{4 \pi R_{\mathrm{s}}^{2} v_{\mathrm{w}} m_{\mathrm{p}}} \\
& =8.2 \times 10^{10} \mathrm{~cm}^{-3}\left(\frac{\dot{M}}{10^{-6} M_{\odot} \mathrm{yr}^{-1}}\right)\left(\frac{R_{\mathrm{s}}}{50 R_{\odot}}\right)^{-2}\left(\frac{v_{\mathrm{w}}}{30 \mathrm{~km} \mathrm{~s}^{-1}}\right)^{-1} .
\end{aligned}
$$

The termination shock distance depends on the density $n_{\text {ISM }}$ and speed $v_{\text {ISM }}$ of the interstellar medium of the distant galaxy for which reasonable assumptions can be made. We note that $v_{\text {ISM }}$ is a relative speed between the interstellar medium and the star, while the speed of the star (cloud) used in our simulation is the speed of the star (cloud) penetrating the jet. From hydrodynamical considerations, the termination shock distance $R_{\mathrm{s}, \mathrm{t}}$ can be calculated as (Parker 1958; Wilkin 2000)

$$
\begin{aligned}
R_{\mathrm{s}, \mathrm{t}}= & \sqrt{\frac{\dot{M} v_{\mathrm{w}}}{4 \pi m_{\mathrm{p}} n_{\mathrm{ISM}} v_{\mathrm{ISM}}^{2}}} \\
= & 3.1 \times 10^{16} \mathrm{~cm}\left(\frac{\dot{M}}{10^{-6} M_{\odot} \mathrm{yr}^{-1}}\right)^{1 / 2}\left(\frac{v_{\mathrm{w}}}{30 \mathrm{~km} \mathrm{~s}^{-1}}\right)^{1 / 2} \\
& \times\left(\frac{n_{\mathrm{ISM}}}{100 \mathrm{~cm}^{-3}}\right)^{-1 / 2}\left(\frac{v_{\mathrm{ISM}}}{100 \mathrm{~km} \mathrm{~s}^{-1}}\right)^{-1}
\end{aligned}
$$

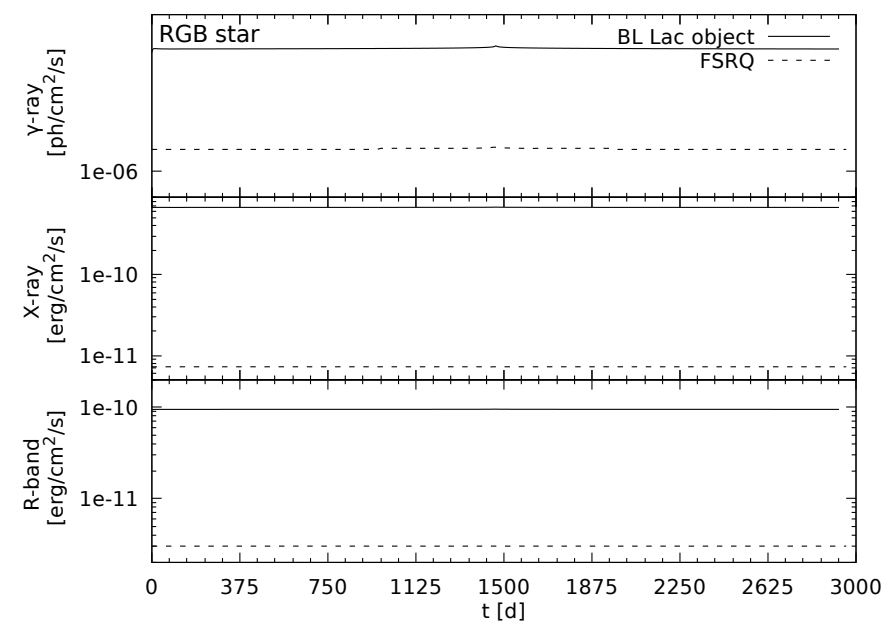

Fig. A.1. Lightcurves for the FSRQ (dashed line) and BL Lac object (solid line) cases in the observer's frame for three energy bands for the astrosphere of an RGB star. See text for parameters. We note the logarithmic $y$-axes.

which corresponds to about $2000 \mathrm{AU}$. Using $r_{\mathrm{s}, 0}=0.5 \mathrm{AU}$ provides the result shown in Fig. A.1.

The mass loss rate $\dot{M}$ used in Eqs. (A.1) and (A.2) is at the upper end of RGB mass loss rates, which may be achieved only during a short period of time close to the end of the RGB phase of the star. It is important to note that a reduction in $\dot{M}$ reduces both the central density and the termination shock distance.

Therefore, the result shown in Fig. A.1 provides an upper limit case on what could be achieved in this scenario. Apparently, the astrosphere does not contain enough particles to provide a meaningful flare. While a very minor variation is visible in the center of the lightcurve, this tiny variation is not observable within the usual fluctuations of blazar lightcurves. The presence of numerous stars in the jet at the same time (Araudo et al. 2013; Vieyro et al. 2017) may provide sufficient particles to achieve a more pronounced flare.

Wolf-Rayet stars exhibit even greater mass loss rates than RGB stars with $\dot{M}_{\mathrm{WR}} \sim 10^{-4}$. They might provide sufficiently dense winds in order to produce meaningful flares. However, their total number in a galaxy are very low, and the odds of one interacting with the jet are even lower. 